\title{
Quarkonium Suppression from Coherent Energy Loss in Fixed-Target Experiments Using LHC Beams
}

\author{
François Arleo ${ }^{1}$ and Stéphane Peigné ${ }^{2}$ \\ ${ }^{1}$ Laboratoire Leprince-Ringuet (LLR), École Polytechnique, CNRS/IN2P3, 91128 Palaiseau, France \\ ${ }^{2}$ SUBATECH, UMR 6457, Université de Nantes, Ecole des Mines de Nantes, IN2P3/CNRS, 4 rue Alfred Kastler, \\ 44307 Nantes Cedex 3, France
}

Correspondence should be addressed to François Arleo; francois.arleo@cern.ch

Received 3 May 2015; Accepted 26 July 2015

Academic Editor: Cédric Lorcé

Copyright (c) 2015 F. Arleo and S. Peigné. This is an open access article distributed under the Creative Commons Attribution License, which permits unrestricted use, distribution, and reproduction in any medium, provided the original work is properly cited. The publication of this article was funded by SCOAP S $^{3}$

Quarkonium production in proton-nucleus collisions is a powerful tool to disentangle cold nuclear matter effects. A model based on coherent energy loss is able to explain the available quarkonium suppression data in a broad range of rapidities, from fixed-target to collider energies, suggesting coherent energy loss in cold nuclear matter to be the dominant effect in quarkonium suppression in p-A collisions. This could be further tested in a high-energy fixed-target experiment using a proton or nucleus beam. The nuclear modification factors of $\mathrm{J} / \psi$ and $\Upsilon$ as a function of rapidity are computed in $\mathrm{p}$-A collisions at $\sqrt{s}=114.6 \mathrm{GeV}$, and in $\mathrm{p}-\mathrm{Pb}$ and $\mathrm{Pb}-\mathrm{Pb}$ collisions at $\sqrt{s}=72 \mathrm{GeV}$. These center-of-mass energies correspond to the collision on fixed-target nuclei of $7 \mathrm{TeV}$ protons and $2.76 \mathrm{TeV}$ (per nucleon) lead nuclei available at the LHC.

\section{Introduction}

Understanding the physical origin of quarkonium $(J / \psi, \Upsilon)$ suppression in proton-nucleus ( $\mathrm{p}-\mathrm{A})$ collisions has been a challenge for the past thirty years. This would of course be a prerequisite in order to perform reliable baseline predictions in heavy-ion collisions, where quarkonia are expected to be dissociated due to Debye screening of the heavy-quark potential at finite temperature [1]. Perhaps even more importantly, the wealth of data (especially for $J / \psi$ and $\Upsilon$ ) available in $\mathrm{p}$-A collisions could help to understand generic features of hard QCD processes in a nuclear environment.

In everyday language, we often make the distinction between "fixed-target" and "collider" experiments when it comes to quarkonium production. This separation might look a bit artificial but not entirely:

(i) In fixed-target experiments, luminosities are often high, leading to abundant yields and consequently reduced statistical uncertainties. Moreover, thanks to the boost of the center-of-mass frame of the collision, the rapidity coverage of such experiments can extend up to larger negative values of rapidity (we follow the usual convention where positive (negative) rapidities correspond to the proton (nucleus) fragmentation region) or Feynman- $x, x_{\mathrm{F}} \simeq 2 M_{\perp} / \sqrt{s} \times \sinh y$, where $M_{\perp}$ is the quarkonium transverse mass. The highest energies ever reached are of course rather modest, $\sqrt{s}=38.7 \mathrm{GeV}$ and $\sqrt{s}=41.6 \mathrm{GeV}$, using, respectively, $800 \mathrm{GeV}$ and $920 \mathrm{GeV}$ proton beams at the Tevatron and at HERA. This, however, allows for probing also larger positive values of $x_{\mathrm{F}}$ than in collider experiments.

(ii) At collider energies-RHIC and LHC, to quote only the facilities accelerating heavy ions-unprecedented energies can be reached, respectively, $\sqrt{s}=200 \mathrm{GeV}$ and $\sqrt{s}=5.02 \mathrm{TeV}$, for instance, making the production of $\Upsilon$ states, marginally measured in fixedtarget experiments, easier. In terms of acceptance, quarkonia are detected in a narrow window in $x_{\mathrm{F}}$, centered around $x_{\mathrm{F}}=0$.

Let us illustrate this with a few examples, starting with one of the first experiments which measured $J / \psi$ suppression in 
p-A collisions. The NA3 spectrometer at the CERN SPS collected 1.5 million $\mathrm{J} / \psi$ events (!) in hadron-nucleus collisions [2], allowing for precise measurements close to the kinematic edge of phase-space, $x_{\mathrm{F}} \lesssim 0.75$ (on the contrary, the coverage at RHIC and LHC is, resp., $\left|x_{\mathrm{F}}\right| \lesssim 0.2$ and $\left|x_{\mathrm{F}}\right| \lesssim 0.02$ for $\mathrm{J} / \psi$ production). It is remarkable that these data, taken in the early 1980s, prove to be as competitive as the most recent $\mathrm{LHC}$ results when it comes to understanding $\mathrm{J} / \psi$ suppression in nuclei. More than a decade after NA3, the FNAL E866 experiment reported on high-statistics measurements of $\mathrm{J} / \psi$ $\left(3 \times 10^{6}\right.$ events $)$ and $\psi^{\prime}\left(10^{5}\right)$ production on several nuclear targets in the range $-0.2 \lesssim x_{\mathrm{F}} \lesssim 0.9$ [3]. Other important measurements were reported by experiments at the SPS (NA38 [4], NA50 [5], and NA60 [6]) and HERA (HERA-B [7]), yet on a more restricted $x_{\mathrm{F}}$ range.

These data are nicely supplemented by those carried out in d-Au collisions at RHIC (PHENIX $[8,9]$ and STAR [10]) and in $\mathrm{p}-\mathrm{Pb}$ collisions at LHC (ALICE [11] and LHCb [12]). At LHC, the relative suppression of $\Upsilon$ excited states $(2 \mathrm{~S}, 3 \mathrm{~S})$ with respect to $1 S$ states has been performed by CMS [13], not to mention open heavy-flavor data (D mesons in ALICE [14], $\mathrm{B}$ mesons in CMS [15], and nonprompt $\mathrm{J} / \psi$ coming from $\mathrm{B}$ decays in ALICE [16] and LHCb [12]).

Several cold nuclear matter (CNM) effects could in principle affect quarkonium yields in proton-nucleus collisions. Without being comprehensive, let us mention the following ones:

(i) Quarkonia may interact inelastically with the surrounding nucleons they may encounter while propagating through the nucleus. Such nuclear absorption may happen when the quarkonium formation time (in the rest frame of the nucleus) is comparable or less than the medium length $L, \tau_{f} \times \cosh y_{\text {lab }} \lesssim L$, where $\tau_{f}$ is the proper formation time $\left(\tau_{f} \simeq 0.3 \mathrm{fm}\right.$ for both $\mathrm{J} / \psi$ and $\Upsilon$ ) and $y_{\text {lab }}$ is the quarkonium rapidity in the nucleus rest frame. Note that $y_{\text {lab }}$ is directly related to the momentum fraction $x_{2}$ carried by the target parton, cosh $y_{\text {lab }}=M_{\perp} /\left(2 m_{p} x_{2}\right)$, where $m_{p}$ is the proton mass.

(ii) Parton distribution functions (PDF) are known to be different in a proton and in a nucleus at all values of $x$. Such nuclear PDF (nPDF) effects could either suppress or enhance quarkonium yields in $\mathrm{p}-\mathrm{A}$ collisions (with respect to $\mathrm{p}-\mathrm{p}$ collisions) depending on the value of the momentum fraction $x_{2}$. When $x_{2}$ is small (typically when the time for the hard process to occur is large in the nucleus rest frame, $t_{\mathrm{h}} \simeq\left(1 / M_{\perp}\right) \times \cosh y_{\text {lab }}=1 /\left(2 m_{p} x_{2}\right) \gtrsim L$, using $L=10 \mathrm{fm}$, this would correspond to $x_{2} \leqslant 10^{-2}$ ), the nucleons in the nucleus act coherently leading to a reduction of the quarkonium yield in a nucleuscalled shadowing [17], or saturation [18] to use a more modern language-as compared to the incoherent sum over $A$ independent nucleons.

(iii) Nuclear transverse momentum broadening of the heavy-quark pair induces coherent gluon radiation, arising from the interference between emission amplitudes off the initial projectile parton and the final color octet quark pair. This coherent mediuminduced radiation leads to an average induced energy loss proportional to the quarkonium energy [19]. The consequences of coherent energy loss are quarkonium suppression (resp., enhancement) at large positive (resp., large negative) values of the rapidity and at all center-of-mass energies of the $\mathrm{p}-\mathrm{A}$ collision.

Although each of these CNM effects is plausible, it does not necessarily mean that all play a role in the nuclear dependence of quarkonium production; in particular, the strength of each CNM effect is usually unknown a priori. A sound strategy is to investigate each of these effects separately, through a systematic and quantitative comparison to all available data, while keeping the smallest number of assumptions and free parameters.

Quarkonium suppression reported at forward rapidities cannot be reproduced by either nuclear absorption or nPDF effects, nor by a mixture of both. Although the comparison to RHIC and LHC data only may still give the impression that strong $\mathrm{nPDF}$ effects could explain $\mathrm{J} / \psi$ data [11] (for examples of $\mathrm{nPDF}$ effects on quarkonium production in $\mathrm{p}-\mathrm{Pb}$ collisions at LHC, see $[20,21])$, the significant suppression measured by the fixed-target experiments (NA3 and E866) on a wider $x_{\mathrm{F}}$ range is clearly incompatible with the predictions of these two effects. An elegant way to be persuaded is to plot $\mathrm{J} / \psi$ suppression data as a function of $x_{2}=M_{\perp} / \sqrt{s} \times \exp (-y)$ [22]. The suppression from either nuclear absorption or nPDF effects is expected to be a function of $x_{2}$ only, independent of $\sqrt{s}$. This is in strong disagreement with the accumulated data from fixed-target and RHIC experiments (see $[3,23]$ ). Without a doubt, the world data indicate that at least another cold nuclear matter effect is at play.

Contrary to nuclear absorption or nPDF effects, the sole effect of coherent energy loss is able to reproduce the data on quarkonium suppression, from fixed-target to collider energies [24-26]. Detailed comparisons were published elsewhere, so let us only highlight the phenomenological successes of this approach:

(i) The $x_{\mathrm{F}}$ (or $y$ ) dependence of $\mathrm{J} / \psi$ suppression is well reproduced on a very large domain (up to large values of $x_{\mathrm{F}} \leqslant 0.8$, when data are available) and at all centerof-mass energies, from $\sqrt{s} \simeq 20 \mathrm{GeV}$ to $\sqrt{s}=5 \mathrm{TeV}$.

(ii) The $p_{\perp}$ dependence is well-reproduced too, either at a fixed-target experiment (E866) or at colliders (RHIC, LHC), although the $p_{\perp}$ dependence seems slightly more abrupt in the model than in collider data. The centrality dependence measured by PHENIX at RHIC is also nicely described.

(iii) $\Upsilon$ measurements in p-A collisions are compatible with the expected mass dependence of coherent energy loss, although the present experimental uncertainties are still fairly large.

(iv) Finally, an original prediction of coherent energy loss is a different magnitude of quarkonium suppression in $\mathrm{p}-\mathrm{A}$ and $\pi$-A collisions (in contrast with nuclear absorption effects, which should be independent of 
the projectile hadron), in agreement with the measurements of NA3.

The strength of coherent energy loss depends on a single free parameter, namely, the magnitude of the cold nuclear matter transport coefficient, $\widehat{q}_{0}=0.075 \mathrm{GeV}^{2} / \mathrm{fm}$ at $x=10^{-2}$, obtained from a fit of the precise E866 measurements in p-W collisions.

We find it appealing that the variety of quarkonium measurements in $\mathrm{p}-\mathrm{A}$ collisions can be described using a single CNM effect. Of course, by no means does this imply that no other CNM effects could play a role too, yet these clearly appear to be subleading when the quarkonium is produced at "large enough" rapidity. Both nPDF and coherent energy loss effects could in principle be incorporated consistently in the picture. As a matter of fact, attempts have been made in [25]. However, given the large theoretical uncertainties on nuclear parton distributions, due to the lack of small- $x$ measurements in nuclear collisions (in this respect a high-energy electronion collider would be highly beneficial for the community. Let us mention in passing that no coherent energy loss effects are expected in deep-inelastic scattering experiments as the incoming projectile particle is color neutral [19]), especially for gluon densities at small $x$, we prefer to focus on the single (but in our opinion, leading) effect of coherent energy loss for which rather precise calculation can be performed.

An exciting possibility to further constrain cold nuclear matter effects (on quarkonium production, but not only) would be to smash the LHC proton and lead beams on a collection of fixed nuclear targets [27]. We believe that this proposal would combine the above-discussed advantages of fixed-target and collider experiments.

In this paper, the predictions for quarkonium suppression due to coherent energy loss in $\mathrm{p}$-A collisions at $\sqrt{\mathrm{s}}=$ 114.6 GeV (corresponding to the nominal $7 \mathrm{TeV}$ proton beam energy at the LHC) and $\mathrm{Pb}-\mathrm{A}$ collisions at $\sqrt{s}=72 \mathrm{GeV}$ (corresponding to the $2.76 \mathrm{TeV}$ lead beam) are given. Before this, we recall in the next section the main ingredients of our approach.

\section{Coherent Energy Loss Model in a Nutshell}

2.1. Formulation. We briefly detail in this section the basics of the model based on coherent energy loss used to describe $\psi$ (denoting $\mathrm{J} / \psi$ or $\Upsilon$ ) suppression measured in proton-nucleus collisions. (The model can also be formulated in heavy-ion (A-B) collision; see [28] for details.) The single differential pA production cross section as a function of the $\psi$ energy reads [25]

$$
\frac{1}{A} \frac{\mathrm{d} \sigma_{\mathrm{pA}}^{\psi}}{\mathrm{d} E}(E)=\int_{0}^{\varepsilon^{\max }} \mathrm{d} \varepsilon \mathscr{P}\left(\varepsilon, E, \ell_{\mathrm{A}}^{2}\right) \frac{\mathrm{d} \sigma_{\mathrm{pp}}^{\psi}}{\mathrm{d} E}(E+\varepsilon),
$$

where $E$ (resp., $\varepsilon$ ) is the energy (resp., energy loss) of the $Q \bar{Q}$ pair in the rest frame of the nucleus $A$. The upper limit on the energy loss is $\varepsilon^{\max }=\min \left(E, E_{\mathrm{p}}-E\right)$, where $E_{\mathrm{p}}$ is the beam energy in that frame. $\mathscr{P}$ denotes the energy loss probability distribution, or quenching weight.

The quenching weight is related to the medium-induced, coherent radiation spectrum $\mathrm{d} I / \mathrm{d} \varepsilon$ given in [25] (and earlier
TABle 1: Values of $L$ used in $\mathrm{p}, \mathrm{Ca}, \mathrm{Cu}$, and $\mathrm{Pb}$ targets.

\begin{tabular}{lcccc}
\hline Nucleus & $\mathrm{p}$ & $\mathrm{Ca}$ & $\mathrm{Cu}$ & $\mathrm{Pb}$ \\
\hline Atomic mass & 1 & 40 & 63 & 208 \\
$L(\mathrm{fm})$ & 1.5 & 5.69 & 6.67 & 10.11 \\
\hline
\end{tabular}

in [19]), which is a very good approximation to the exact spectrum computed to all orders in the opacity expansion [29]. It depends on the accumulated transverse momentum transfer $\ell_{\mathrm{A}}=\sqrt{\widehat{q} L}$ (assumed to satisfy $\ell_{\mathrm{A}} \ll M_{\perp}$ ) due to soft rescatterings in the nucleus, where $L$ is the medium pathlength and $\hat{q}$ is the transport coefficient in cold nuclear matter (in our picture, the relevant transverse broadening $\sqrt{\hat{q} L}$ is that of the compact color octet crossing the nucleus, and $L$ is thus the path-length from the "front" surface to the "back" surface of the nucleus for a given impact parameter. Note that, in nuclear absorption models, the relevant length is that from the hard production vertex to the "back" surface of the nucleus, that is, a factor $\sim 2$ smaller than the medium length $L$ used here and quoted in Table 1). More precisely [25],

$$
\begin{aligned}
\widehat{q} & \equiv \widehat{q}_{0}\left[\frac{10^{-2}}{\min \left(x_{0}, x_{2}\right)}\right]^{0.3} ; \\
x_{0} & \equiv \frac{1}{2 m_{\mathrm{p}} L} ; \\
x_{2} & \equiv \frac{M_{\perp}}{\sqrt{s}} e^{-y},
\end{aligned}
$$

where $y$ is the quarkonium rapidity in the center-of-mass frame of the proton-nucleon collision.

Using the fact that the quenching weight is a scaling function of the variable $\varepsilon / E$, namely, $E \mathscr{P}\left(\varepsilon, E, \ell^{2}\right)=\widehat{\mathscr{P}}\left(\varepsilon / E, \ell^{2}\right)$, we can rewrite (1) as [28]

$$
\begin{aligned}
\frac{1}{A} & \frac{\mathrm{d} \sigma_{\mathrm{pA}}^{\psi}}{\mathrm{d} y}(y, \sqrt{s})=\int_{0}^{\delta y^{\max }(y)} \mathrm{d} \delta y \widehat{\mathscr{P}}\left(e^{\delta y}-1, \widehat{q}(y) L\right) \\
& \frac{\mathrm{d} \sigma_{\mathrm{pp}}^{\psi}}{\mathrm{d} y}(y+\delta y, \sqrt{s}) .
\end{aligned}
$$

Here, $\delta y^{\max }(y)=\min \left(\ln 2, y_{\max }-y\right)$, with $y_{\max }=\ln \left(\sqrt{s} / M_{\perp}\right)$ the maximal $\psi$ rapidity (in the proton-nucleon c.m. frame) allowed by kinematics. Using (3), we can determine the nuclear modification factor in $\mathrm{p}-\mathrm{A}$ collision:

$$
R_{\mathrm{pA}}^{\psi}(y, \sqrt{s})=\frac{(1 / A)\left(\mathrm{d} \sigma_{\mathrm{pA}}^{\psi} / \mathrm{d} y\right)(y, \sqrt{s})}{\left(\mathrm{d} \sigma_{\mathrm{pp}}^{\psi} / \mathrm{d} y\right)(y, \sqrt{s})} .
$$

As mentioned in the introduction, quarkonium may suffer inelastic interaction with the surrounding nucleons while escaping the nucleus. Although we do not aim to include such an effect in the present predictions, we nevertheless indicate the critical rapidity $y^{\text {crit }}$ :

$$
y^{\mathrm{crit}}(\sqrt{s}, L) \equiv \ln \left(\frac{L}{\tau_{f}} \cdot \frac{2 m_{\mathrm{p}}}{\sqrt{s}}\right)
$$

below which nuclear absorption might come into play. 


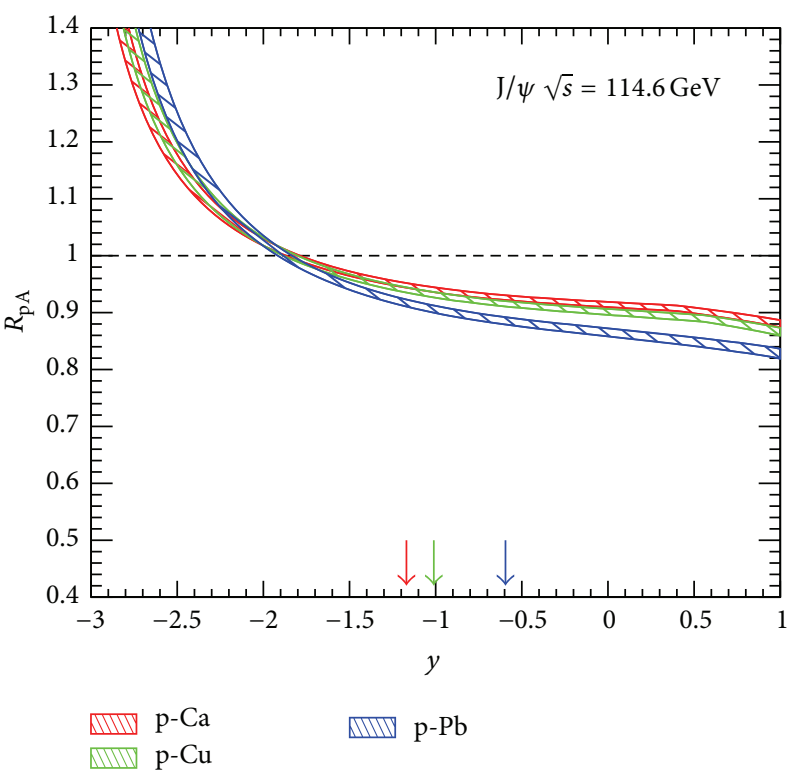

(a)

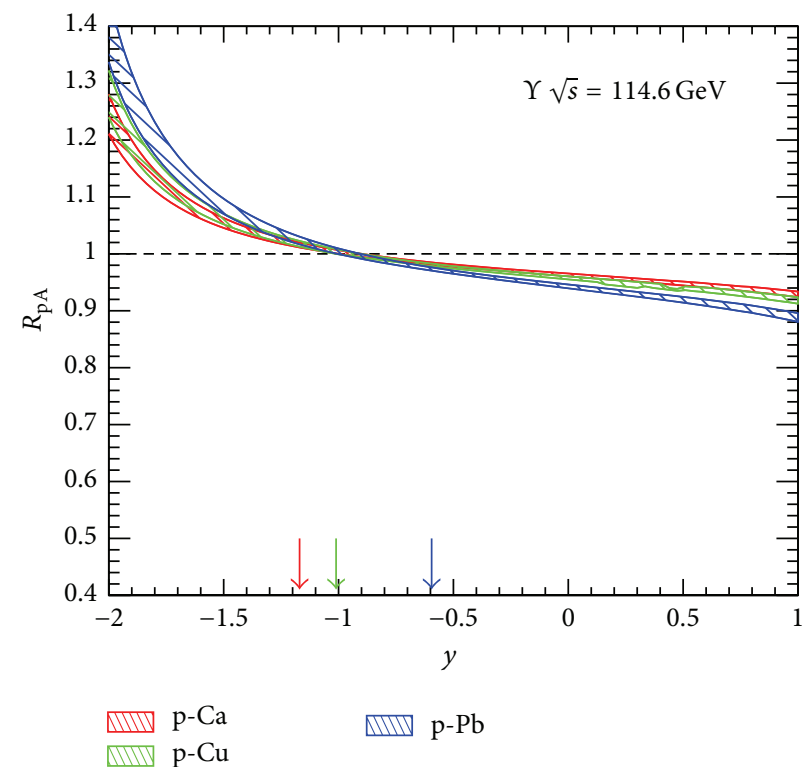

(b)

Figure 1: J/ $\psi$ (a) and $\Upsilon$ (b) suppression in $\mathrm{p}-\mathrm{Ca}, \mathrm{p}-\mathrm{Cu}$, and $\mathrm{p}-\mathrm{Pb}$ collisions at $\sqrt{s}=114.6 \mathrm{GeV}$.

2.2. Ingredients. The medium length $L$ is obtained from a Glauber model calculation using realistic nuclear densities. The values are given in [25] and reproduced in Table 1 for the nuclei of interest in the present paper. In addition, (3) requires the knowledge of the p-p cross section. It is given by a simple parameterization $\mathrm{d} \sigma_{\mathrm{pp}}^{\psi} / \mathrm{d} y \propto(1-$ $\left.\left(2 M_{\perp} / \sqrt{s}\right) \cosh y\right)^{n(\sqrt{s})}$, where the exponent $n$ is obtained from a fit to $\mathrm{p}$-p measurements. Lacking p-p data at the energies of interest $(\sqrt{s}=114.6 \mathrm{GeV}$ in $\mathrm{p}-\mathrm{A}$ and $\sqrt{s}=72 \mathrm{GeV}$ in Pb-A collisions), an interpolation between the values obtained at FNAL $(\sqrt{s}=38.7 \mathrm{GeV})$ and RHIC $(\sqrt{s}=200 \mathrm{GeV})$ energies has been performed. The exponents used in the present paper are given in Table 2. Note that the normalization of the $\mathrm{p}-\mathrm{p}$ cross section is irrelevant here as it cancels out when computing (4).

The transport coefficient $\widehat{q}_{0}$ is the only free parameter of the model. It is determined by fitting the $J / \psi$ suppression measured by E866 [3] in p-W over p-Be collisions $(\sqrt{s}=38.7 \mathrm{GeV})$; see [25]. The obtained value is $\widehat{q}_{0}=$ $0.075_{-0.005}^{+0.015} \mathrm{GeV}^{2} / \mathrm{fm}$.

\section{Results}

3.1. $p-A$ Mode. The predictions for $\mathrm{J} / \psi$ and $\Upsilon$ suppression in $\mathrm{p}-\mathrm{Ca}, \mathrm{p}-\mathrm{Cu}$, and $\mathrm{p}-\mathrm{Pb}$ collisions at $\sqrt{s}=114.6 \mathrm{GeV}$ are shown in Figure 1. The rapidity range is chosen to match the acceptance of detectors like LHCb [30]. In terms of Feynman$x$, the range $-3<y<1$ (resp., $-2<y<1$ ) corresponds to $-0.54<x_{\mathrm{F}}<0.06$ (resp., $-0.60<x_{\mathrm{F}}<0.19$ ) for $\mathrm{J} / \psi$ (resp., $\Upsilon$ (because of the more restricted phase-space from its larger mass, the rapidity acceptance is smaller for $\Upsilon$ than for $\mathrm{J} / \psi)$ ).

The $J / \psi$ suppression is rather moderate, less than $20 \%$, and does not vary too strongly with rapidity except at
TABLE 2: Values of $n$ used at $\sqrt{s}=72 \mathrm{GeV}$ and $\sqrt{s}=114.6 \mathrm{GeV}$ for $\mathrm{J} / \psi$ and $\Upsilon$.

\begin{tabular}{lcc}
\hline Mode & Pb-A & p-A \\
\hline$\sqrt{s}(\mathrm{GeV})$ & 72 & 114.6 \\
$n_{\mathrm{J} / \psi}$ & $5.1 \pm 0.2$ & $6.0 \pm 0.3$ \\
$n_{\Upsilon}$ & $4.1 \pm 0.3$ & $5.0 \pm 0.4$ \\
\hline
\end{tabular}

very negative rapidity values, $y<y_{0} \simeq-2$, where $\mathrm{J} / \psi$ enhancement $\left(R_{\mathrm{pA}}>1\right)$ can be seen. In this rapidity region, however, nuclear absorption may come into play as can be seen from the vertical arrows indicating the values of $y^{\text {crit }}$ $\left(y^{\text {crit }} \simeq-1\right)$ for each target. The suppression depends on the atomic mass number of the target $(\mathrm{Ca}, \mathrm{Cu}$, and $\mathrm{Pb})$, yet this nuclear dependence is tempered somehow by the slow dependence of the coherent energy loss scale, $\widehat{\omega}$, on the medium length, $\widehat{\omega} \propto \sqrt{L}$ [25].

The shape of $Y$ suppression is similar. The value of the rapidity at which $R_{\mathrm{pA}}\left(y_{0}\right)=1$ is $y_{0} \simeq-1$, that is, one more unit than in the $J / \psi$ channel. This can be understood from the approximate $x_{\mathrm{F}}$ scaling present in the model [25] (at a given rapidity $y$, the corresponding value of $x_{\mathrm{F}}$ is larger for $\Upsilon$ than for $\mathrm{J} / \psi$ due to the larger transverse mass, $x_{\mathrm{F}} \propto M_{\perp}$ ) which would predict the difference between these two "crossing points" to be $y_{0}^{\Upsilon}-y_{0}^{\mathrm{J} / \psi} \sim \ln \left(M^{\mathrm{Y}} / M^{\mathrm{J} / \psi}\right) \simeq 1$.1. Once more, nuclear absorption may affect $\Upsilon$ suppression, though maybe not as much as the $J / \psi$ because of its smaller radius.

3.2. A-p Mode. Let us move now to calculations corresponding to an incoming $2.76 \mathrm{TeV} \mathrm{Pb}$ beam on a proton and $\mathrm{a} \mathrm{Pb}$ target, shown in Figure 2. This configuration allows for probing more easily quarkonium suppression in the 


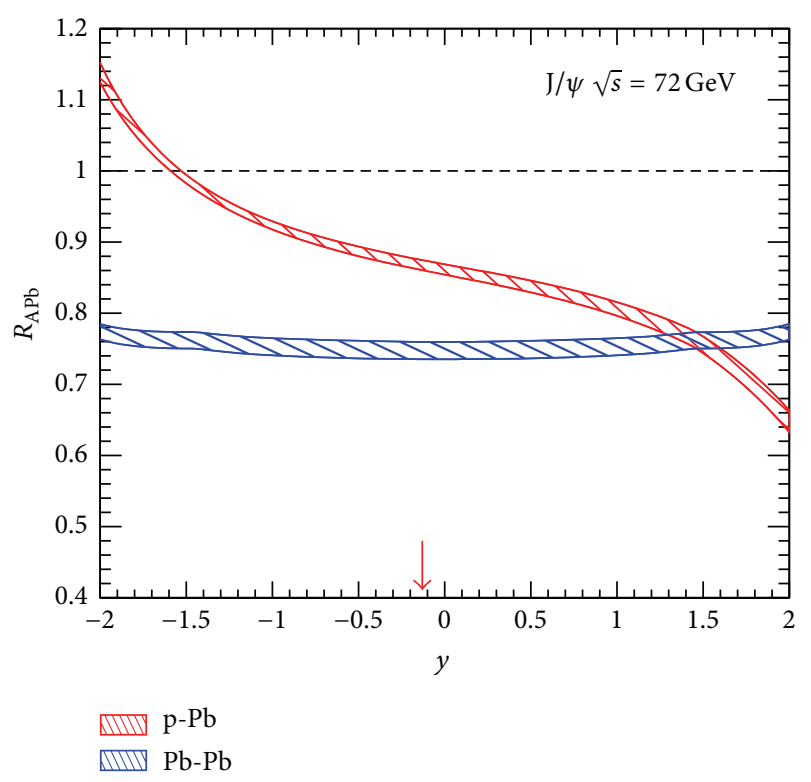

(a)

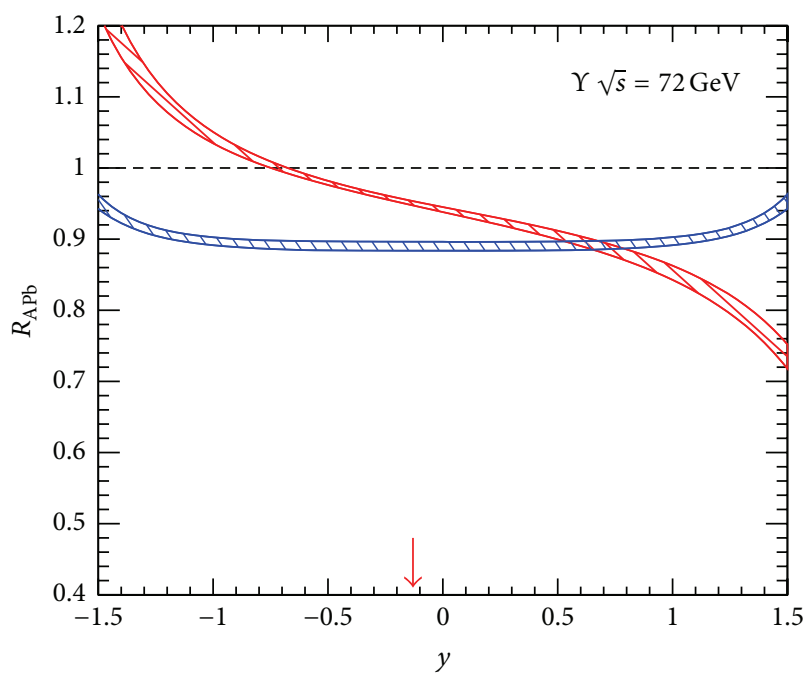

IIIV $\mathrm{p}-\mathrm{Pb}$

IIIV $\mathrm{Pb}-\mathrm{Pb}$

(b)

Figure 2: $\mathrm{J} / \psi$ (a) and $\Upsilon(\mathrm{b})$ suppression in $\mathrm{p}-\mathrm{Pb}$ and $\mathrm{Pb}-\mathrm{Pb}$ collisions at $\sqrt{s}=72 \mathrm{GeV}$.

proton fragmentation, that is, at positive rapidities. (Although the $\mathrm{Pb}$ nucleus collides with a proton, we will keep the convention that positive values of $y$ correspond to the proton fragmentation region.) The chosen rapidity range $-2<y<2$ (resp., $-1.5<y<1.5$ ) corresponds to $-0.31<x_{\mathrm{F}}<0.31$ $\left(-0.56<x_{\mathrm{F}}<0.56\right)$ for $\mathrm{J} / \psi(\Upsilon)$. The lower center-ofmass energy however shifts $y^{\text {crit }}$ in $\mathrm{p}-\mathrm{Pb}$ collisions towards larger values, possibly leading to more pronounced nuclear absorption.

In $\mathrm{Pb}-\mathrm{Pb}$ collisions, the suppression is naturally an even function of $y$. In such collisions, one expects a hot medium to be formed leading to extra quarkonium suppression. Therefore, the results should rather be seen as baseline calculations than genuine predictions. Moreover, in A-A collisions, the condition for hadronization taking place outside both nuclei reads $y^{\text {crit }}<y<-y^{\text {crit }}$. This condition is only met in $\mathrm{Pb}$ $\mathrm{Pb}$ collisions at $\sqrt{s}=72 \mathrm{GeV}$ around midrapidity, $|y| \lesssim 0.1$. At larger $|y|$, the quarkonium state will be fully formed in one of the two nuclei and thus possibly sensitive to nuclear absorption.

\section{Conflict of Interests}

The authors declare that there is no conflict of interests regarding the publication of this paper.

\section{References}

[1] T. Matsui and H. Satz, " $J / \psi$ suppression by quark-gluon plasma formation," Physics Letters B, vol. 178, pp. 416-422, 1986.

[2] J. Badier, J. Boucrot, J. Bourotte et al., "Experimental $J / \psi$ hadronic production from 150 to $280 \mathrm{GeV} / c$," Zeitschrift für Physik C, vol. 20, pp. 101-116, 1983.

[3] M. J. Leitch, W. M. Lee, M. E. Beddo et al., "Measurement of differences between $J / \psi$ and $\psi^{\prime}$ suppression in $p-A$ collisions," Physical Review Letters, vol. 84, no. 15, pp. 3256-3260, 2000.

[4] M. C. Abreu, C. Baglin, A. Baldit et al., "Charmonia production in $450 \mathrm{GeV} / \mathrm{c}$ proton induced reactions," Physics Letters B, vol. 444, pp. 516-522, 1998.

[5] B. Alessandro, C. Alexa, R. Arnaldi et al., "J/ $\psi$ and $\psi^{\prime}$ production and their normal nuclear absorption in proton-nucleus collisions at $400 \mathrm{GeV}$," The European Physical Journal C, vol. 48, no. 2, pp. 329-341, 2006.

[6] R. Arnaldi, K. Banicz, J. Castor et al., " $J / \psi$ production in protonnucleus collisions at 158 and $400 \mathrm{GeV}$,' Physics Letters B, vol. 706, no. 4-5, pp. 263-267, 2012.

[7] I. Abt, M. Adams, M. Agari et al., "Kinematic distributions and nuclear effects of $J / \psi$ production in $920 \mathrm{GeV}$ fixed-target proton-nucleus collisions," The European Physical Journal C, vol. 60, no. 4, pp. 525-542, 2009.

[8] A. Adare, S. Afanasiev, C. Aidala et al., "Cold nuclear matter effects on $J / \psi$ yields as a function of rapidity and nuclear geometry in $d+A$ collisions at $\sqrt{s_{N N}}=200 \mathrm{GeV}$," Physical Review Letters, vol. 107, Article ID 142301, 2011.

[9] A. Adare, S. Afanasiev, C. Aidala et al., “Transverse-momentum dependence of the $J / \psi$ nuclear modification in $d+$ Au collisions at $\sqrt{s_{N N}}=200 \mathrm{GeV}$,' Physical Review C, vol. 87, Article ID 034904, 2013.

[10] L. Adamczyk, J. K. Adkins, G. Agakishiev et al., "Suppression of $\Upsilon$ production in $\mathrm{d}+\mathrm{Au}$ and $\mathrm{Au}+\mathrm{Au}$ collisions at $\sqrt{s_{N N}}=200$ GeV," Physics Letters B, vol. 735, pp. 127-137, 2014.

[11] B. B. Abelev, J. Adam, D. Adamová et al., "J/ $/ \psi$ production and nuclear effects in $\mathrm{p}-\mathrm{Pb}$ collisions at $\sqrt{\mathrm{s}_{\mathrm{NN}}}=5.02 \mathrm{TeV}$," Journal of High Energy Physics, vol. 2014, no. 2, article 073, 2014.

[12] R. Aaij, B. Adeva, M. Adinolfi et al., "Study of $J / \psi$ production and cold nuclear matter effects in $\mathrm{p} \mathrm{Pb}$ collisions at $\sqrt{s_{N N}}=5$ TeV," Journal of High Energy Physics, vol. 2014, no. 2, article 072, 2014.

[13] S. Chatrchyan, V. Khachatryan, A. M. Sirunyan et al., "Event activity dependence of $Y(\mathrm{nS})$ production in $\sqrt{s_{N N}}=5.02 \mathrm{TeV}$ $\mathrm{pPb}$ and $\sqrt{s}=2.76 \mathrm{TeV}$ pp collisions," Journal of High Energy Physics, vol. 2014, no. 4, article 103, 2014. 
[14] B. B. Abelev, J. Adam, M. M. Aggarwal et al., "Measurement of prompt $D$-meson production in $p$-Pb Collisions at $\sqrt{s_{N N}}=$ 5.02 TeV," Physical Review Letters, vol. 113, no. 23, Article ID 232301, 11 pages, 2014.

[15] CMS Collaboration, "Measurements of the $B^{+}, B^{0}$ and $B_{s}^{0}$ production cross sections in pPb collisions at $\sqrt{s_{\mathrm{NN}}}=5.02 \mathrm{TeV}$," Tech. Rep. CMS-PAS-HIN-14-004, CERN, 2014.

[16] J. Adam, D. Adamova, M. M. Aggarwal et al., "Inclusive, prompt and non-prompt $J / \psi$ production at mid-rapidity in $\mathrm{Pb}-\mathrm{Pb}$ collisions at $\sqrt{s_{N N}}=2.76 \mathrm{TeV}$,' Journal of High Energy Physics, vol. 2015, no. 7, article 051, 2015.

[17] N. Armesto, "Nuclear shadowing," Journal of Physics G: Nuclear and Particle Physics, vol. 32, no. 11, p. R367, 2006.

[18] F. Gelis, E. Iancu, J. Jalilian-Marian, and R. Venugopalan, “The color glass condensate," Annual Review of Nuclear and Particle Science, vol. 60, pp. 463-489, 2010.

[19] F. Arleo, S. Peigné, and T. Sami, "Revisiting scaling properties of medium-induced gluon radiation," Physical Review D, vol. 83, Article ID 114036, 2011.

[20] J. Albacete, N. Armesto, R. Baier et al. et al., "Predictions for $p+\mathrm{Pb}$ collisions at $\sqrt{s_{N N}}=5 \mathrm{TeV}$," International Journal of Modern Physics E, vol. 22, Article ID 1330007, 2013.

[21] E. Ferreiro, F. Fleuret, J. Lansberg, and A. Rakotozafindrabe, "Impact of the nuclear modification of the gluon densities on $J / \psi$ production in $p \mathrm{~Pb}$ collisions at $\sqrt{s_{N N}}=5 \mathrm{TeV}$," Physical Review C, vol. 88, Article ID 047901, 2013.

[22] P. Hoyer, M. Vanttinen, and U. Sukhatme, "Violation of factorization in charm hadroproduction," Physics Letters B, vol. 246, no. 1-2, pp. 217-220, 1990.

[23] M. J. Leitch, "Overview of charm physics at RHIC," AIP Conference Proceedings, vol. 892, no. 1, pp. 404-409, 2007.

[24] F. Arleo and S. Peigné, " $J / \psi$ suppression in $p-A$ collisions from parton energy loss in cold QCD matter," Physical Review Letters, vol. 10, no. 12, Article ID 122301, 5 pages, 2012.

[25] F. Arleo and S. Peigné, "Heavy-quarkonium suppression in p-A collisions from parton energy loss in cold QCD matter," Journal of High Energy Physics, vol. 2013, no. 3, article 122, 2013.

[26] F. Arleo, R. Kolevatov, S. Peigné, and M. Rustamova, "Centrality and $p_{\perp}$ dependence of $J / \psi$ suppression in proton-nucleus collisions from parton energy loss," Journal of High Energy Physics, vol. 2013, no. 5, article 155, 2013.

[27] S. J. Brodsky, F. Fleuret, C. Hadjidakis, and J. P. Lansberg, "Physics opportunities of a fixed-target experiment using LHC beams," Physics Reports, vol. 522, no. 4, pp. 239-255, 2013.

[28] F. Arleo and S. Peigné, "Quarkonium suppression in heavy-ion collisions from coherent energy loss in cold nuclear matter," Journal of High Energy Physics, vol. 2014, no. 10, article 73, 2014.

[29] S. Peigné, F. Arleo, and R. Kolevatov, "Medium-induced gluon radiation: an update," http://arxiv.org/abs/1402.1671.

[30] L. Massacrier, B. Trzeciak, F. Fleuret et al., "Feasibility studies for quarkonium production at a fixed-target experiment using the LHC proton and lead beams (AFTER@LHC)," http://arxiv.org/abs/1504.05145. 

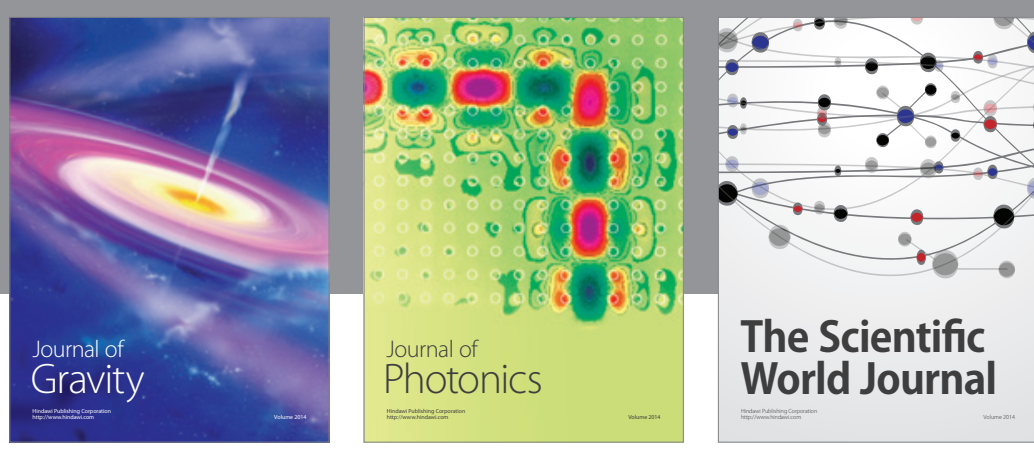

The Scientific World Journal
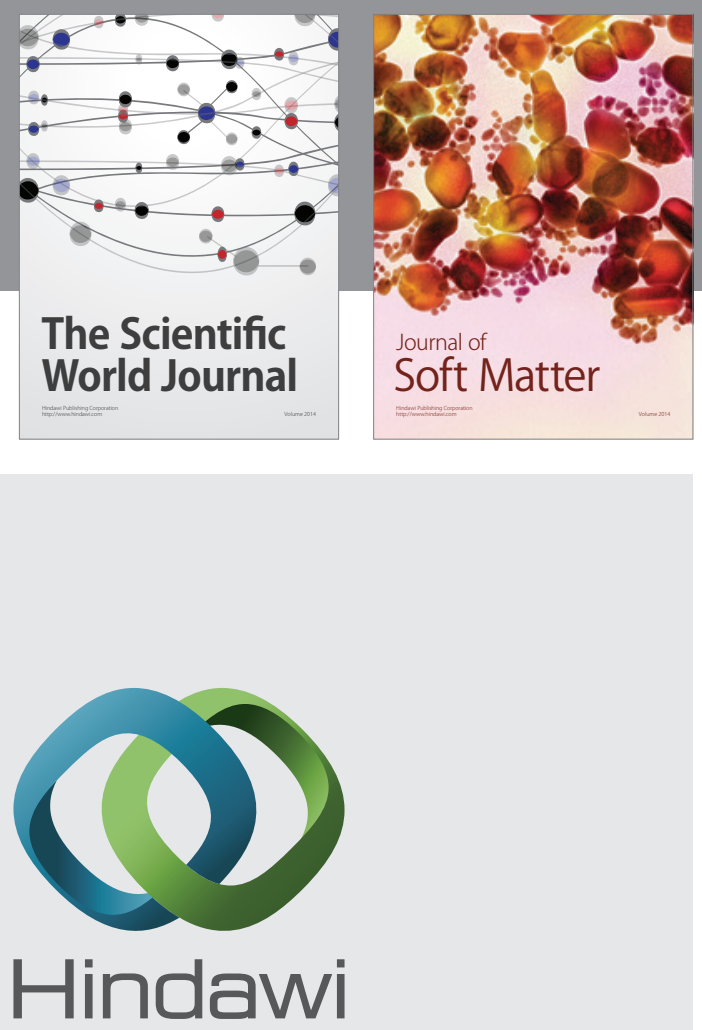

Submit your manuscripts at

http://www.hindawi.com

nternational Journal of

Statistical Mechanics
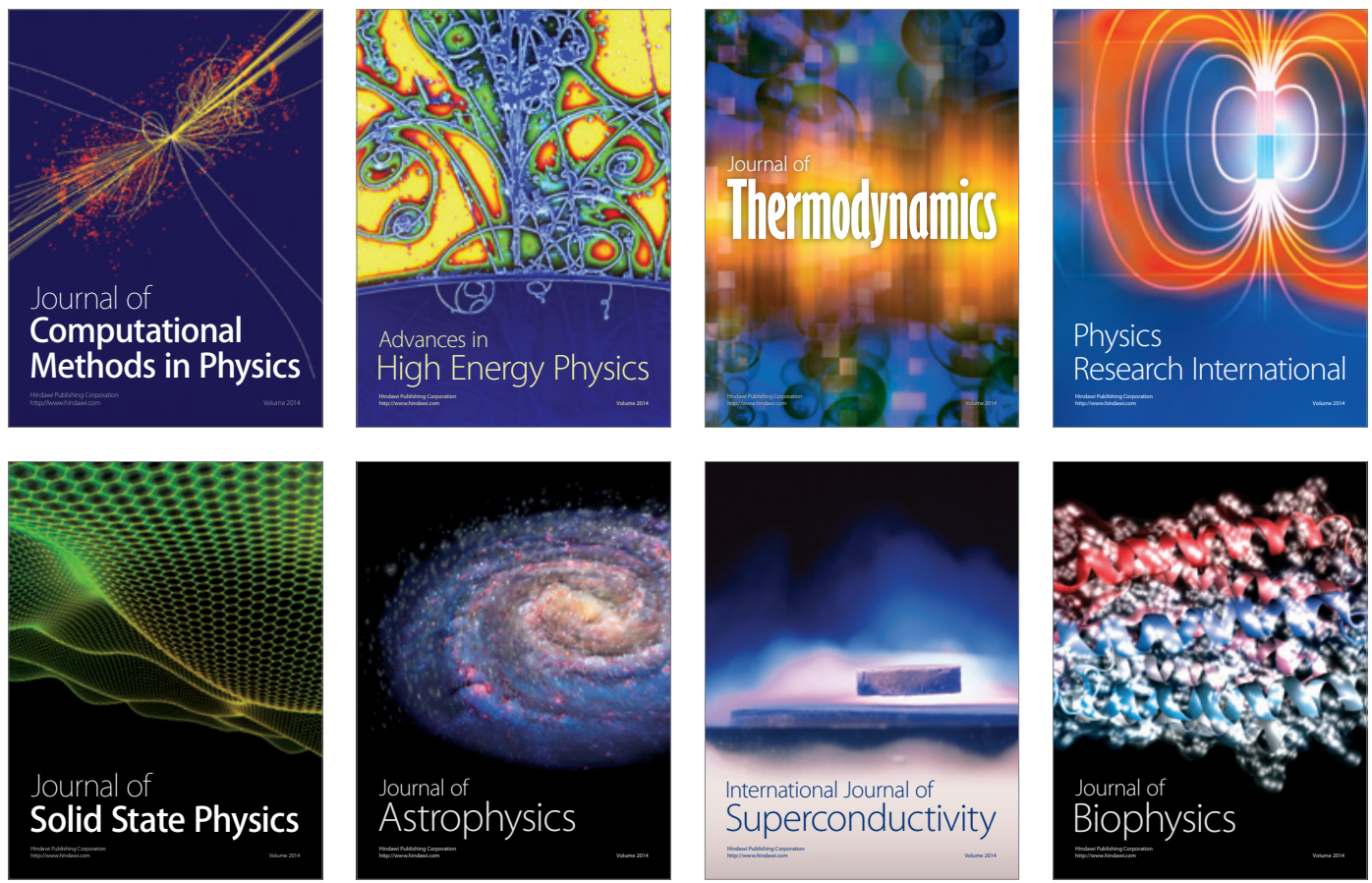
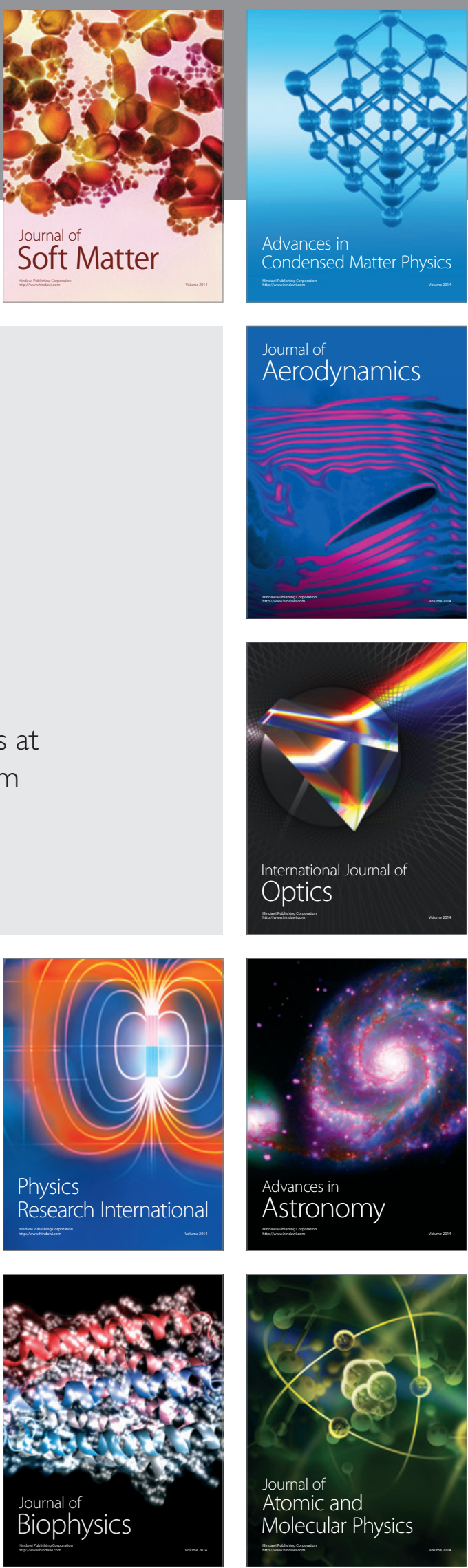